\title{
A Photoactivatable Probe for Super-Resolution Imaging of Enzymatic Activity in Live Cells
}

\section{Journal Article}

\section{Author(s):}

Halabi, Elias A.; Thiel, Zacharias; Trapp, Nils; Pinotsi, Dorothea; Rivera-Fuentes, Pablo

Publication date:

2017-09-20

Permanent link:

https://doi.org/10.3929/ethz-b-000192479

\section{Rights / license:}

In Copyright - Non-Commercial Use Permitted

\section{Originally published in:}

Journal of the American Chemical Society 139(37), https://doi.org/10.1021/jacs.7b07748 


\title{
A Photoactivatable Probe for Super-Resolution Imaging of Enzymatic Activity in Live Cells
}

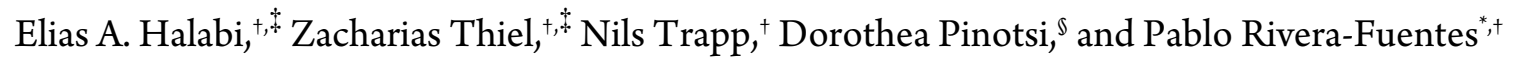 \\ ${ }^{\dagger}$ Laboratory of Organic Chemistry, Vladimir-Prelog-Weg 3, and ${ }^{\S}$ Scientific Center for Optical and Electron Microscopy, Otto-Stern- \\ Weg 3, ETH Zurich, CH-8093, Zurich, Switzerland.
}

\begin{abstract}
A dual-activatable, fluorogenic probe was developed to sense esterase activity with single-molecule resolution. Without enzymatic pre-activation, the diazoindanone-based probe has an electron poor core and, upon irradiation, undergoes Wolff rearrangement to give a ring-expanded xanthene core that is non-emissive. If the probe is pre-activated by carboxylesterases, the tricyclic core becomes electronrich and the photoinduced Wolff rearrangement produces a highly emissive rhodol dye. Live-cell and solution studies confirmed the selectivity of the probe and revealed that the photoactivated dye does not diffuse away from the original location of activation because the intermediate ketene forms a covalent bond with surrounding macromolecules. Single-molecule localization microscopy was used to reconstruct a super-resolved image of esterase activity. These single-molecule images of enzymatic activity changed significantly upon treatment of the cells with inhibitors of human carboxylesterase I and II, both in terms of total number of signals and intracellular distribution. This proof-of-principle study introduces a sensing mechanism for single-molecule detection of enzymatic activity that could be applied to many other biologically relevant targets.
\end{abstract}

\section{INTRODUCTION}

Light microscopy has revolutionized the study of living systems but its classical resolution is limited by diffraction of light. This limit is proportional to the wavelength of light divided by twice the numerical aperture of the objective (resolved distance $>200 \mathrm{~nm}$ ). ${ }^{1}$ Several strategies have been developed to break this barrier and their implementation achieves super-resolution imaging of nanometric cellular structures. ${ }^{2}$ Stochastic optical reconstruction microscopy (STORM) ${ }^{3}$ and photoactivated localization microscopy (PALM) ${ }^{4}$ are closely related methods that achieve single-molecule resolution by sequential activation, readout and deactivation of photochemically active fluorophores. Many photoactive fluorescent proteins ${ }^{5}$ and small-molecule dyes ${ }^{6}$ have been developed for STORM and PALM. These emitters have facilitated the localization and tracking of macromolecules in live specimens, ${ }^{7,8}$ enhancing the resolution of optical microscopy well beyond the limit of diffraction. Despite these advances, sensing and quantification of biochemical activity with nanometer resolution is a challenge that remains largely unmet because current photoactivatable fluorophores are not responsive to specific enzymes.

Fluorescent sensors are molecules that respond to a stimulus by exhibiting a measurable change in their photophysical properties. ${ }^{9} \mathrm{~A}$ variety of protein-based and small-molecule sensors have been developed to detect metal ions, ${ }^{10,11}$ small signaling molecules, ${ }^{12} \mathrm{pH},{ }^{13}$ and redox states, ${ }^{14}$ among many other stimuli. These probes have been applied in diffraction-limited microscopy with great success, but sensors that are specifically designed for application in super-resolution microscopy have only started to appear very recently. ${ }^{15}$ To the best of our knowledge, there are no small-molecule sensors that can be directly applied to STORM/PALM imaging, because these techniques require photoactivatable fluorophores and the sensing mechanism must be coupled to the photochemical conversion. Herein, we address this challenge by developing a dual enzyme- and photo-activatable probe that provides super-resolved subcellular imaging of the activity of esterases, which are important enzymes involved in detoxification of xenobiotics and pro-drug activation. ${ }^{16}$ We further demonstrate that besides producing super-resolved images, single-molecule localization data collected with this probe can be used to obtain dynamic information and quantify the subcellular localization of microdomains of enzymatic activity.

\section{RESULTS AND DISCUSSION}

Design and synthesis. Belov, Wurm, Hell, and co-workers reported that xanthene dyes can be locked in a dark isomer upon conversion of the lactone into diazoindanones. ${ }^{17}$ These molecules undergo Wolff rearrangement upon photolysis, which restores the conjugation in the xanthene core (Scheme 1A). The diazoindanone photoremovable group is a small, non-polar modification well suited for live-cell superresolution imaging. ${ }^{18}$ More recently, Lavis and co-workers observed that diazoindanone derivatives of silicon rhodamines undergo Wolff rearrangement to give primarily a ring expansion product that is only very weakly fluorescent. ${ }^{19}$ Silicon xanthenes are more electrophilic than oxygen-substituted xanthenes, ${ }^{20}$ making them more prone to attack by the putative carbene intermediate of the Wolff rearrangement to give a dark, seven-membered ring photoproduct (Scheme 1A). We envisioned that this differential reactivity could be harnessed as a sensing mechanism. As proof-of-principle, we designed the electron-poor probe 1, which should photoisomerize to a dark, seven-membered ring photoproduct (Scheme 1B). Upon removal of the acetyl group by carboxylesterases, compound 2 is obtained, which is electron-rich and 
should photoisomerize to give a bright rhodol emitter (Scheme 1B). Such probe would effectively be a fluorescent probe for endogenous esterase activity and could be employed in single-molecule localization imaging, as we show below.

Scheme 1. A) Transformations of oxygen and silicon rhodamines protected with a diazoindanone photoremovable group. B) Proposed mechanism of enzyme sensing and photoactivation using a substituted, rhodol-based diazoindanone.

A
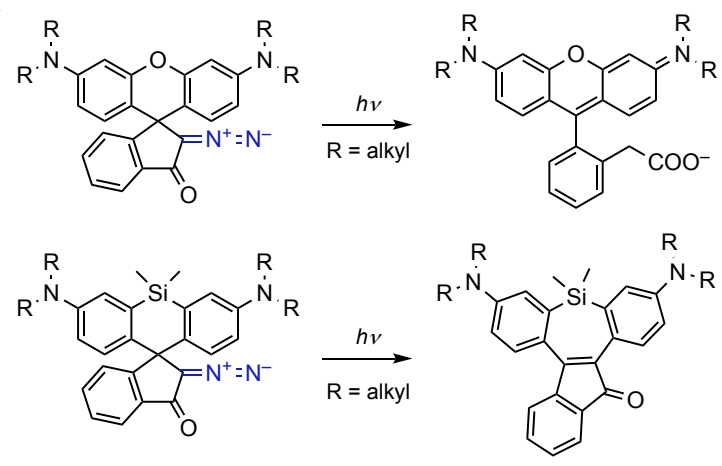

B
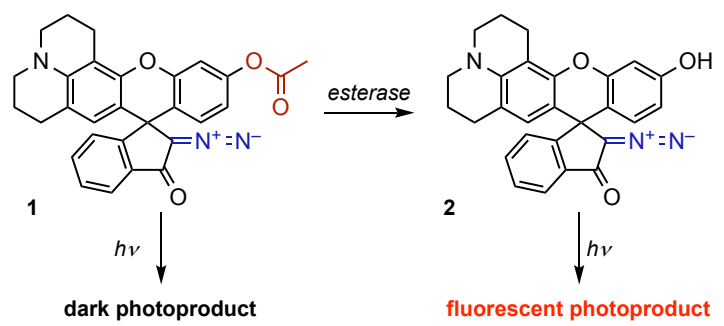

Compound 1 was synthesized in two steps (Scheme 2) starting from rhodol 3. ${ }^{21}$ Treatment of this compound with acetic anhydride and pyridine gave the acetylated rhodol 4 . The diazoindanone group was formed by conversion of the lactone into the acid chloride and treatment with (trimethylsilyl)diazomethane. Deprotection of 1 was achieved with $\mathrm{NaOH}$ to yield deacetylated product 2 .

Scheme 2. Synthesis of compounds 1 and 2. DIPEA = diisopropylethylamine; TMS = trimethylsilyl.
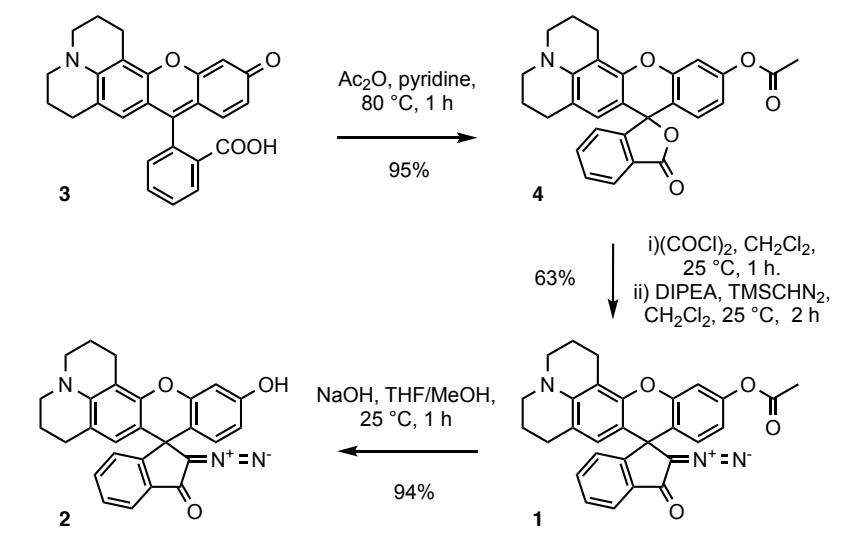

Photochemistry and photophysics. The photoinduced Wolff rearrangements of 1 and 2 were investigated in buffered solutions ( 0.25 $\mu \mathrm{M}$, phosphate-buffered saline, $\mathrm{pH}=7.4)$. The samples were irradiated with UV light $(\sim 300 \mathrm{~nm}$, for details see Materials and Methods $)$ and fluorescence emission was recorded at defined time intervals (Figure 1A). The increase in fluorescence signal plateaued after 25 min for both photoreactions. Whereas a significant increase in fluorescence was detected in the photolysis of 2 , only a small change was measured for compound 1 , indicating that acetylation of the phenolic group suppresses the formation of emissive species 


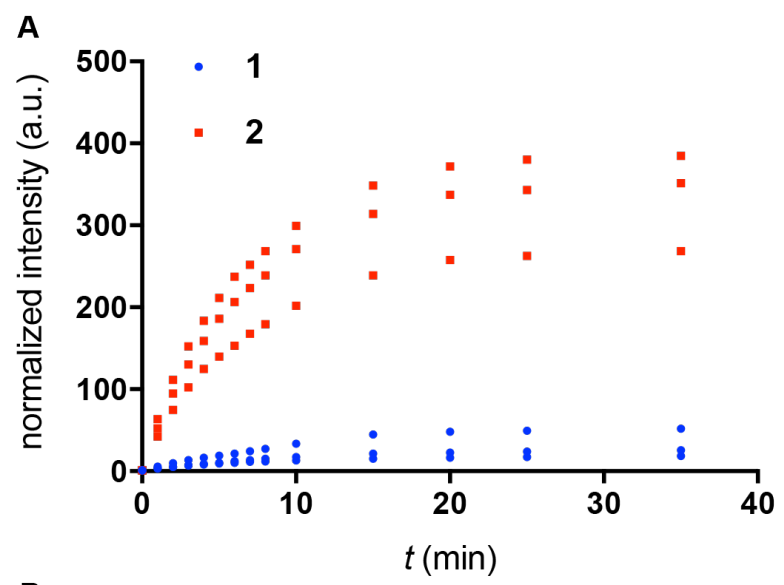

B
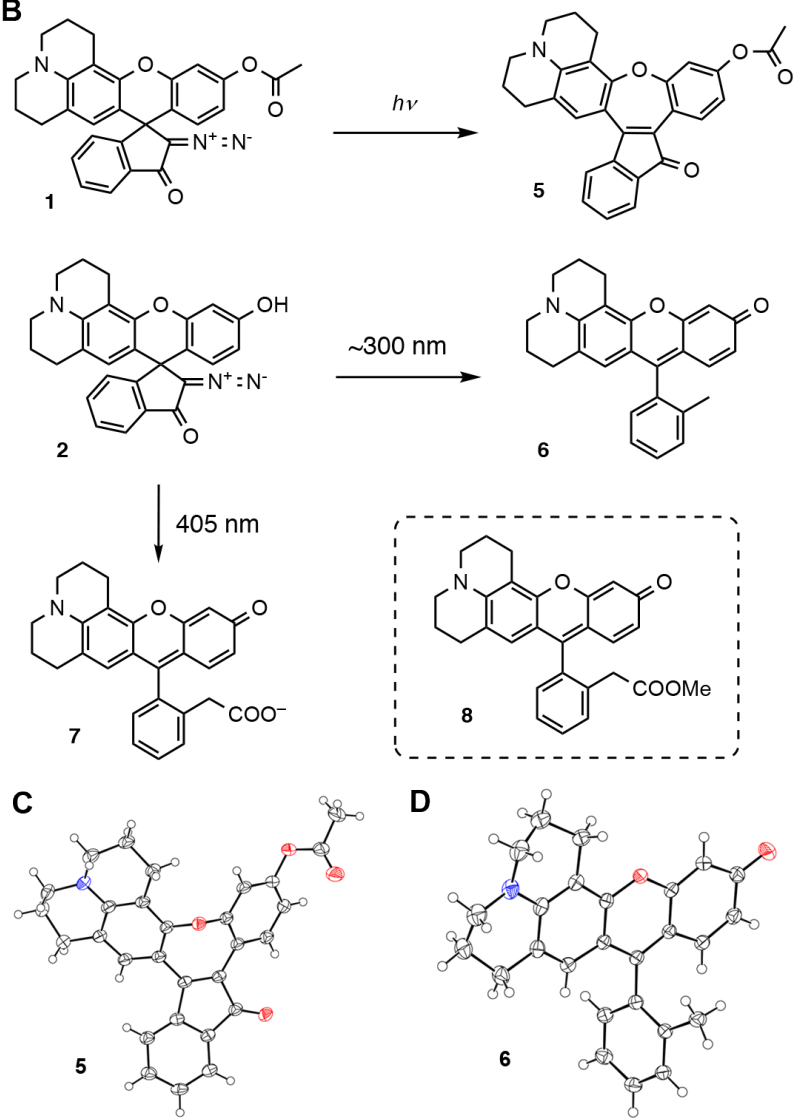

Figure 1. A) Fluorescence increase upon irradiation $(\sim 300 \mathrm{~nm})$ of solutions of 1 and $2(0.25 \mu \mathrm{M}$, phosphate-buffered saline, $\mathrm{pH}=7.4$, in quartz cuvettes). Three independent measurements are depicted. B) Main photoreactions of compounds 1 and 2, and structure of compound 8 . C) X-ray structure of photoproduct 5 (CCDC: 1564214). D) X-ray structure of photoproduct 6 (CCDC: 1564215).

. Isolation of the major photoproducts (see the SI) revealed that under these conditions, photolysis of compound 1 yielded predominantly product 5 , whereas compound 2 gave mostly compound 6 (Figure 1B), in which the product of the Wolff rearrangement underwent further decarboxylation. The identification of these compounds was established by spectroscopic methods and single-crystal X-ray diffraction (Figures 1C and 1D). Product 5 displays an absorption maximum $\left(\lambda_{\mathrm{abs}}\right)$ at $565 \mathrm{~nm}$ with an extinction coefficient $(\varepsilon)$ of $3.8 \times 10^{3} \mathrm{M}^{-1} \mathrm{~cm}^{-1}$, and exhibits essentially no fluorescence (Figure S7). In contrast, compound 6 displays $\lambda_{\text {abs }}=541 \mathrm{~nm}, \varepsilon=2.9 \times 10^{4} \mathrm{M}^{-1} \mathrm{~cm}^{-1}$ and an emission quantum yield $\left(\phi_{\mathrm{em}}\right)$ of $36 \%$ (Figure S8). A few other minor photoproducts were isolated from both reactions and some of them characterized (see the SI), but the only considerably emissive photoproducts were isolated from the reaction of compound 2 . This distribution of photoproducts explains the large increase in fluorescence intensity upon irradiation of $\mathbf{2}$ but not 1.Quantum yields of photoactivation were estimated for the disappearance of starting materials 1 and 2 and for the appearance of some photoproducts (Figure S1). Upon irradiation with a $405 \mathrm{~nm}$ LED both compound 1 and 2 were photolyzed with small quantum yields $\left(\phi_{\mathrm{PA}}=0.3 \%\right.$ and $\phi_{\mathrm{PA}}=0.2 \%$, respectively). Under these conditions, the main photoproduct of 1 was compound $5\left(\phi_{\mathrm{PA}}=0.05 \%\right)$ and the main product of 2 was 7 ( $\left.\phi_{\mathrm{PA}}=0.16 \%\right)$. Decarboxylation of compound 7 to give 6 did not occur at $405 \mathrm{~nm}$ (Figure 1B). Preparative amounts of 7 could not be obtained and 
therefore a stable, methyl ester derivative thereof (compound 8, Figure 1B) was prepared independently (see Scheme S3) to estimate the photophysical properties of 7 . The quantum yields and photophysical parameters of all main photoproducts are displayed in Table S1. These experiments confirm that the acetyl group on compound 1 shuts down the formation of emissive rhodol products upon photoinduced Wolff rearrangement. A mechanistic proposal that explains the divergent photochemistry of compounds 1 and 2 is provided in Scheme S4.

Enzymatic and Live-Cell Activation. Compound 1 could be converted into compound 2 by recombinant human carboxylesterase II (hCEII) in solution. Irradiation of samples of $\mathbf{1}$ in the presence of hCEII gave a strong fluorescent signal, whereas a combination of compound 1 , hCEII and $\beta$-lapachone (9, Figure 2D), which is a known inhibitor of carboxylesterases, ${ }^{22}$ gave nearly no change in fluorescence (Figure S19).

The ability of endogenous human esterases to convert compound 1 into 2 within live cells was evaluated by confocal optical microscopy. Cells were incubated with compound 1 at a concentration of $10 \mu \mathrm{M}$, which is lower than the $\mathrm{IC}_{50}$ values determined for this compound, either in the dark $(20 \mu \mathrm{M})$ or upon photoactivation $(17 \mu \mathrm{M}$, Figure S22). After only $10 \mathrm{~min}$ of incubation, photoactivation of 1 with light of $405 \mathrm{~nm}(120 \mathrm{~mW}, 20 \%, 1 \mathrm{~s})$ led to a substantial increase in fluorescence (Figure 2). Pre-treatment of cells with inhibitor 9 (10 $\mu \mathrm{M})$, however, did not decrease significantly the fluorescence after photoactivation (Figure 2C). In contrast, pre-treatment of cells with compound 10 (10 $\mu \mathrm{M}$, Figure 2D), which is an inhibitor of human carboxylesterase I (hCEI), ${ }^{23}$ decreased substantially the fluorescence intensity after photoactivation of compound 1 (Figure 2C). This experiment suggests that either compound 1 is a better substrate of hCEI than hCEII or compound 9 is not an effective inhibitor of hCEII in live cells. In either case, it is clear that the photoactivation of compound 1 depends to a large extent on the activity of at least hCEI and therefore can be used as a fluorescent probe of esterase activity.

Colocalization analysis with various organelle markers revealed that compound $\mathbf{1}$ is activated most efficiently in the endoplasmic reticulum (ER), with some fluorescence being detected also in mitochondria and the Golgi apparatus (for details see Material and Methods, Figure S18 and Table S2).

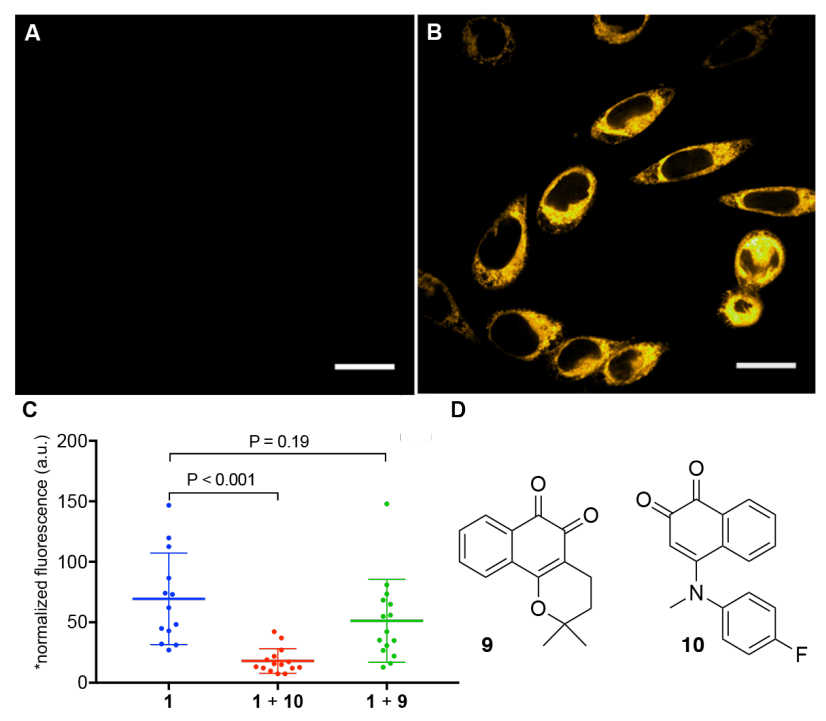

Figure 2. A) HeLa cells imaged at $561 \mathrm{~nm}$ laser excitation after $10 \mathrm{~min}$ of incubation with compound 1 (10 $\mu \mathrm{M})$, before photoactivation. B) Same cells as in panel A after photoactivation $(405 \mathrm{~nm}, 120 \mathrm{~mW}, 20 \%, 1 \mathrm{~s})$. C) *Normalized fluorescence (fluorescence of compound 1 before photoactivation $=1)$ with $561 \mathrm{~nm}$ laser excitation after photoactivation $(405 \mathrm{~nm}, 120 \mathrm{~mW}, 20 \%, 1 \mathrm{~s})$ of HeLa cells incubated with the indicated compound (10 $\mu \mathrm{M}$ of each). 13 to 15 values of independent measurements, mean values and standard deviations are shown. D) Structures of inhibitors 9 and 10 . Scale bars $=10 \mu \mathrm{m}$.

Intracellular Diffusion Dynamics. Determining the precise localization of an enzymatic event with small-molecule fluorescent sensors can be challenging if the activated probe diffuses away of the site of enzymatic reaction before it can be imaged. The Wolff rearrangement of compound 2 (enzymatically activated form of compound $\mathbf{1}$ ) leads to a ketene intermediate that can be trapped by nucleophiles (see synthesis of compound $\mathbf{8}$ in the SI). We envisioned that if photoactivation occurred immediately after compound 1 was converted into compound 2 by an enzyme, the ketene intermediate could be trapped by the esterase itself or at least within its vicinity. To test this hypothesis, we first irradiated a region of interest (ROI, Figure 3A) within a cell that had been pre-treated with compound $\mathbf{1}$ and recorded the fluorescence of the whole cell over a period of $15 \mathrm{~min}$ (Figure 3 and Movie S1). Immediately after photoactivation, there is very fast diffusion of fluorescent molecules to non-activated regions of the cell. This fluorescence, however, is significantly weaker than the fluorescence within the ROI (Figure 3D). A line profile of the fluorescence intensity over the $15 \mathrm{~min}$ period reveals that there is no broadening of the ROI (Figure 3D), indicating that most of the photoactivated molecules stay within the region in which they were converted.

Super-Resolution Imaging. As we showed before, compound 1 can only be photoconverted into a fluorophore after pre-activation by esterases and it does not diffuse from the site of photoactivation. Based on these observations, we concluded that a super-resolved image of intracellular esterase activity could be generated by addition of compound 1 to live cells that were already under constant cycles of irradiation at $405 \mathrm{~nm}$ (photoactivation) and $561 \mathrm{~nm}$ (readout) using highly inclined laminated optical sheet (HILO) ilumination. ${ }^{24}$ We envisioned that 
in such an experiment, molecules of compound 1 would diffuse into the cell and as soon as they react with esterases, they would be rapidly photoconverted, trapped and detected within the vicinity of the enzyme. Single-molecule localization microscopy of those emitters would reveal the intracellular locations of esterase activity.

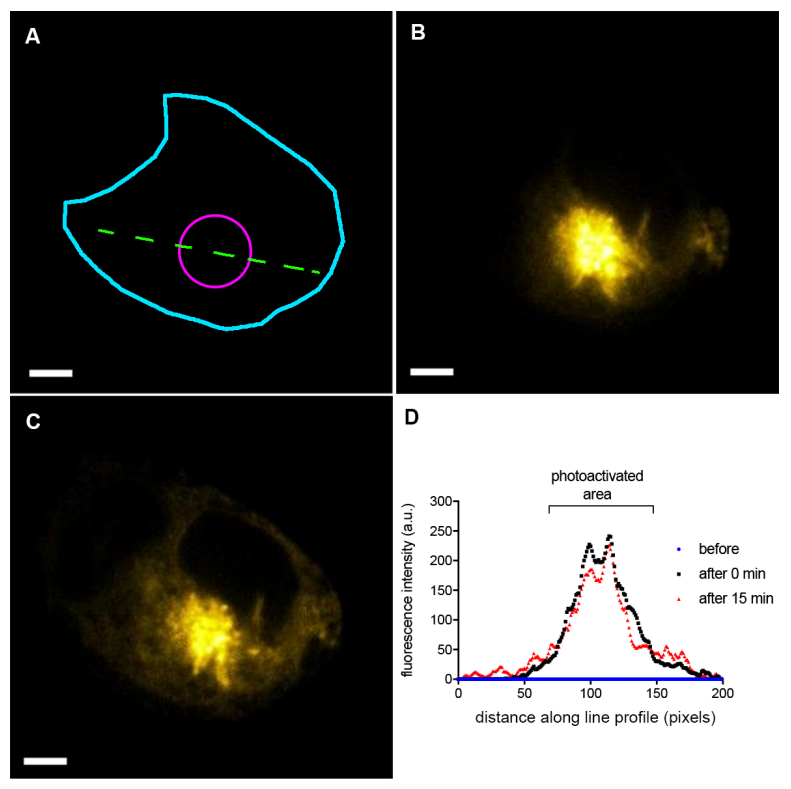

Figure 3. A) HeLa cell imaged with $561 \mathrm{~nm}$ laser excitation after $10 \mathrm{~min}$ of incubation with compound 1 (10 $\mu \mathrm{M})$, before photoactivation. Region of interest (ROI, fuchsia circle), outline of the cell (cyan line), line for intensity profile (dashed green line) are shown. B) Same cell as in panel A, 561 $\mathrm{nm}$ laser excitation, but immediately after photoactivation $(405 \mathrm{~nm}, 120 \mathrm{~mW}, 20 \%, 1 \mathrm{~s})$ within the ROI. C) Same cell as in panel A, $15 \mathrm{~min}$ after photoactivation within the ROI and imaged with $561 \mathrm{~nm}$ laser excitation. D) Line profile (dashed green line in panel A) of the intracellular fluorescence intensity immediately after (black squares) and $15 \mathrm{~min}$ after photoactivation (red triangles). Scale bars $=5 \mu \mathrm{m}$

We performed PALM imaging in HILO mode employing live HeLa cells. An activation pulse of $405 \mathrm{~nm}(100 \mu \mathrm{s}$ duration) was followed by 100 readout camera frames at $561 \mathrm{~nm}$ excitation ( $10 \mathrm{~ms}$ exposure time). This sequence was repeated every second. Compound $1 \mathrm{mas}$ added to the cells approximately $10 \mathrm{~s}$ after the beginning of acquisition and the first single-molecule signals started to appear only a few seconds after addition. A total of 30,000 frames and 360,771 localizations were obtained over a period of $300 \mathrm{~s}$. Using these localizations, a super-resolved image of esterase activity was reconstructed (Figure 4A), with an average localization precision of single molecules of $36 \pm 11 \mathrm{~nm}$ (Figure S23). This image has a much higher resolution than its diffraction-limited ( $>200 \mathrm{~nm}$ ), deconvolved counterpart (Figure 4B). Moreover, acquisition of images under the same conditions, but employing cells that had been pre-treated with inhibitor $10(10 \mu \mathrm{M})$, gave significantly fewer signals than untreated cells (Figure 4C and 4D), confirming that single-molecule signals correspond to esterase activity.

Single-molecule data can be used to extract dynamic information about the sites of activation of compound 1 as it diffuses into the cell. The 30,000 PALM frames ( $5 \mathrm{~min}$ ) were divided in five time intervals of $1 \mathrm{~min}$ each and plotted using different colors for each time interval (Figure $4 \mathrm{E}$ ). Inspection of a magnified section of this time-lapse, color-coded image (Figure 4F) reveals that early localizations are very few and sparsely distributed ( $1 \mathrm{~min}$, blue). As time progresses, activity can be detected in vesicle-like structures (2, 3, and 4 min; green, yellow, and red), and at later time points, the tubular structure of what seems to be the ER starts to become populated ( $5 \mathrm{~min}$, white). 


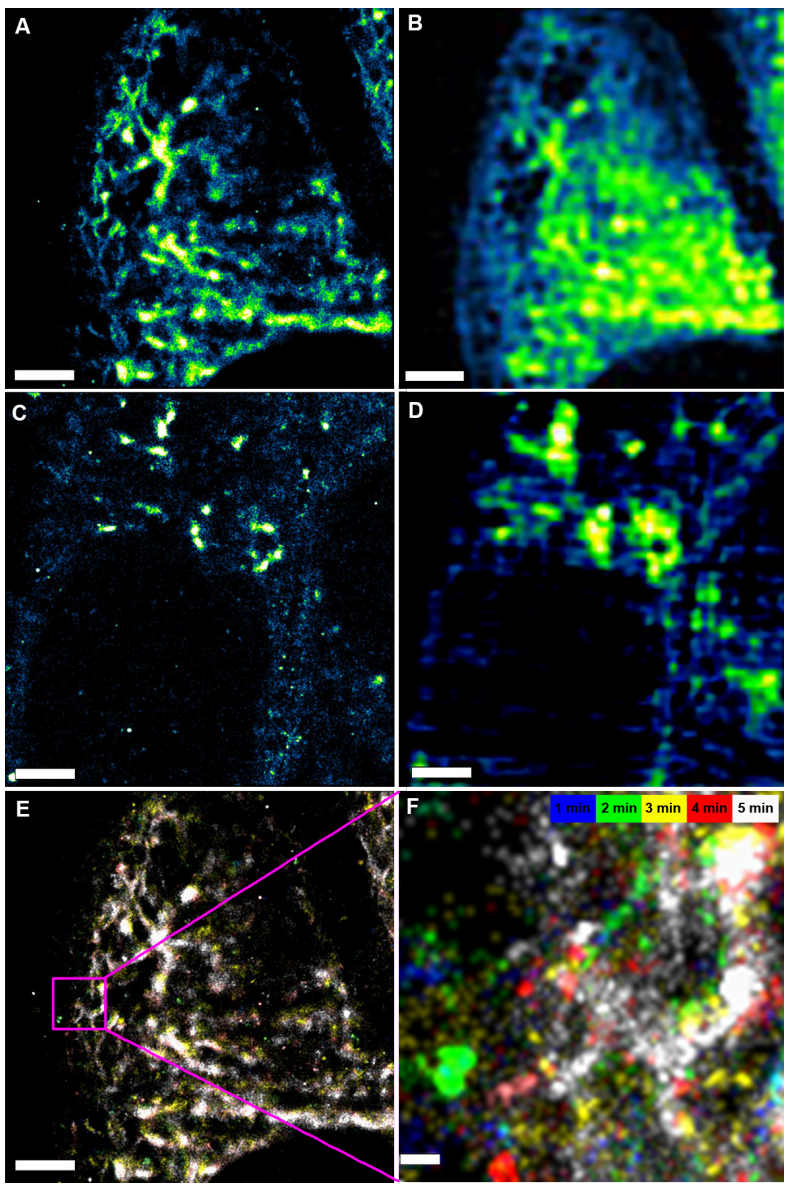

Figure 4. A) PALM super-resolved image of esterase activity in a live HeLa cell treated with compound 1 ( $1 \mu \mathrm{M}, 5$ min acquisition time). B) Diffraction-limited, deconvolved image of the cell shown in panel A. C) PALM super-resolved image of esterase activity in a live HeLa cell treated with compound 1 ( $1 \mu \mathrm{M}, 5 \mathrm{~min}$ acquisition time) and inhibitor $10(10 \mu \mathrm{M}, 30 \mathrm{~min}$ incubation time $)$. D) Diffraction-limited, deconvolved image of the cell shown in panel C. E) Time-lapse, color-coded image of the cell shown in panel A (see color bar in panel F). F) Magnification of the area inside the magenta square in panel E. Scale bars $=3 \mu \mathrm{m}$ (panels A-E); $300 \mathrm{~nm}$ (panel F).

In the experiments described before, it was shown that the fluorescent molecules do not diffuse away from the site of activation and hence represent the distribution of esterase activity. This finding can be further corroborated by single-particle tracking analysis of PALM data (sptPALM).$^{25}$ In sptPALM, single-molecule data can be used to determine which localizations correspond to the same molecule over multiple frames, and such information can be used to reconstruct trajectories of diffusing particles after photoactivation.

Moreover, reconstructed trajectories can be used to calculate the diffusion coefficient $(D)$ of single molecules, allowing us to identify any rapidly diffusing small-molecules $\left(D>20 \mu \mathrm{m}^{2} \mathrm{~s}^{-1}\right)^{26}$ that were not trapped in the vicinity of the activating esterases. 

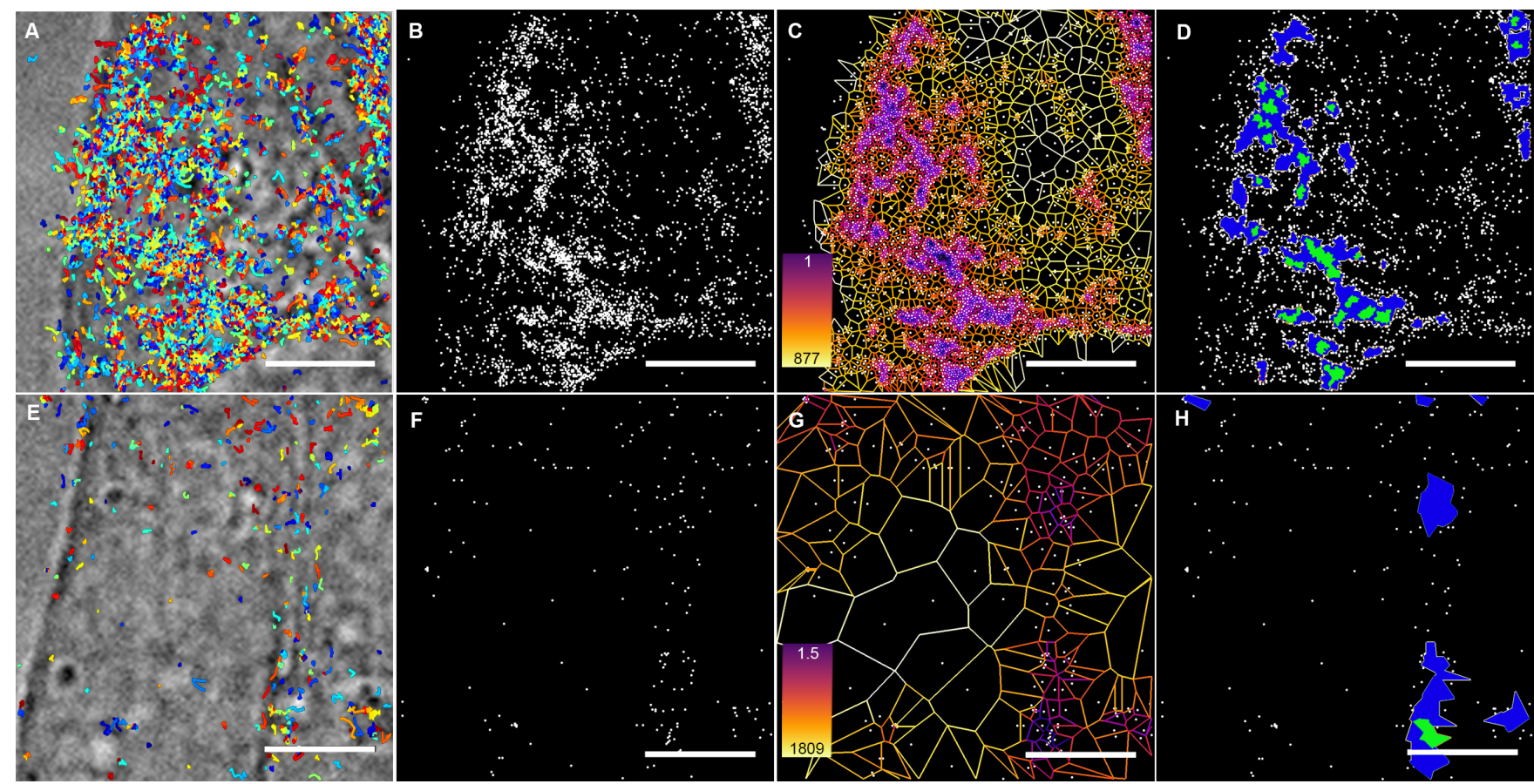

Figure 5. A) Single particle tracks of photoactivated and trapped compound 1, overlaid on a brightfield image of an untreated live HeLa cell (tracks are colored randomly). B) Distribution of track "births" for the cell in panel A. C) Voronoï tessellation of panel B. Polygons are colored according to their areas, see color bar (units: $\mathrm{nm}^{2}$ ). D) Objects (blue) and clusters (green, for definition of objects and clusters see Materials and Methods) identified by Voronoï tessellation in panel C. E) Single particle tracks of photoactivated and trapped compound 1, overlaid on a brightfield image of a HeLa cell that was pre-treated with inhibitor $10(10 \mu \mathrm{M})$. F) Distribution of track "births" for the cell in panel E. G) Voronoï tessellation of panel F. Polygons are colored according to their areas, see color bar (units: $\mathrm{nm}^{2}$ ). H) Objects (green) and clusters (blue) identified by Voronoï tessellation in panel G. Scale bars $=5 \mu \mathrm{m}$. 
To generate single tracks, 20,000 consecutive frames (200 s) were analyzed using DiaTrack ${ }^{27}$ and single-molecule tracks are displayed in Figure 5A. Trajectories with a lifetime smaller than $3 \mathrm{~s}$ and with diffusion coefficients $D>20$ $\mu \mathrm{m}^{2} \mathrm{~s}^{-1}$ were excluded from further analysis. A mean diffusion coefficient of $0.013 \mu \mathrm{m}^{2} \mathrm{~s}^{-1}$ was estimated for the calculated tracks (Figure S24), which confirms that fluorophores are attached to slowly-diffusing, possibly membrane-bound macromolecules. ${ }^{28}$ sptPALM connects multiple signals to obtain the trajectory of a single molecule and, in the present case, intrinsically contains quantitative information about how many molecules of 1 were converted by esterases and photoactivated. Using DiaTrack, we identified the first signal of each track ("birth") and generated an image of single-molecule activations (Figure 5B). An equivalent image was obtained from an experiment in which the cells were treated with inhibitor 10 (Figure 5F, for results with inhibitor 9, see Figure S25). These images show significantly different single-molecule activation events and also very different intracellular distributions. Whereas untreated cells display clusters, cells treated with the inhibitor seem to contain mostly sparsely distributed signals. We quantified the formation of clusters in both images by Voronoï tessellation using SR-Tessler software. ${ }^{29}$ Voronoï tessellation is a segmentation procedure that has been used, for instance, to study the nanoarchitecture of synaptic release of neurotransmitters. ${ }^{30}$ Segmentation of an image of cells treated only with compound 1 identified 22 clusters (Figure 5D, green areas) within regions (objects) of high density of localizations (Figure 5D, blue areas, for definition of clusters and objects, see Materials and Methods). In comparison, segmented images for compound 1 in cells pre-treated with inhibitor 10 yielded only one cluster (Figure 5H, green area, results using inhibitor 9 can be found in Figure S25). Regardless of the total number of localizations, this analysis reveals that clusters of active esterases are lost upon treatment with an inhibitor. This information would have been lost in diffraction-limited microscopy, and we envision that super-resolved sensing could be used to evaluate the ability of inhibitors to reach intracellular microdomains of enzymatic activity, with potential applications in drug discovery. These results demonstrate that quantitative information about the extent and subcellular organization of esterase activity can be extracted from single-molecule localization data employing compound 1 as a super-resolution probe.

\section{SUMMARY AND CONCLUSIONS}

We developed a dual-activatable, small-molecule probe for super-resolved sensing of esterase activity. The sensing mechanism relies on diverging pathways of the photochemical Wolff rearrangement depending on the electronic properties of substituted diazoindanones. We were able to control the photochemical outcome of the reaction by appending an esterase-cleavable substituent that controls the electronic properties of the probe. A combination of solution and live-cell experiments confirmed that the fluorescence response depends on esterase activity and that fluorescent molecules do not diffuse away from their sites of photoactivation. These features allowed us to perform PALM imaging to obtain super-resolved images of esterase activity in live cells. From single-molecule localization data, we identified tracks of single molecules, which led us to confirm that these small-molecule probes do not diffuse substantially from their activation sites. Moreover, the formation of clusters of enzymatic activity could be quantified.

This proof-of-principle study introduced a mechanism for single-molecule localization sensing. The present findings open the path for the development of small-molecule probes to detect, at the nanometer scale, other hydrolytic enzymes, reactive signaling molecules and metal ions.

\section{MATERIALS AND METHODS}

General Methods. All reagents were purchased from commercial sources and used without further purification. Solvents were obtained from Sigma-Aldrich and used as received. NMR spectra were acquired on a Bruker AV300 or Bruker 400 instruments. ${ }^{1} \mathrm{H}$ NMR chemical shifts are reported in ppm relative to $\mathrm{SiMe}_{4}(\delta=0)$ and were referenced internally with respect to residual protons in the solvent $\left(\delta=7.26\right.$ for $\mathrm{CD}_{3} \mathrm{Cl}$ and $\delta=3.31$ for $\left.\mathrm{CD}_{3} \mathrm{OD}\right)$. Coupling constants are reported in $\mathrm{Hz} .{ }^{13} \mathrm{C} \mathrm{NMR}$ chemical shifts are reported in ppm relative to $\mathrm{SiMe}_{4}(\delta=0)$ and were referenced internally with respect to solvent signal $(\delta=$ 77.16 for $\mathrm{CD}_{3} \mathrm{Cl}$ and 49.00 for $\left.\mathrm{CD}_{3} \mathrm{OD}\right)$. Peak assignments are based on calculated chemical shifts, multiplicity and $2 \mathrm{D}$ experiments. IUPAC names of all compounds are provided and were determined using CS ChemBioDrawUltra 15. Highresolution mass spectra (HRMS) were recorded by staff at the Molecular and Bioanalytical (MoBiAS) center (ETH Zurich) employing a Bruker maXis-ESI-Qq-TOF-MS (ESI).

Optical Spectroscopy. Stock solutions in dimethyl sulfoxide (DMSO) were prepared at concentrations of $1 \mathrm{mM}$, stored at $-20{ }^{\circ} \mathrm{C}$ and thawed immediately before each experiment. Spectroscopic measurements were conducted in phosphate-buffered saline (PBS) $\mathrm{pH}=7.4$. UV-Visible spectra were obtained employing a Cary 500 Scan spectrometer using quartz cuvettes from Thorlabs (10 mm path length). Fluorescence spectra were acquired using a Fluorolog 3 fluorimeter (Horiba Jobin-Yvon). All measurements were conducted at room temperature and under red light ambient illumination to avoid photoactivation of the compounds. Extinction coefficients were determined by linear fit of 5 different concentrations of compound in $\mathrm{PBS} / \mathrm{CH}_{3} \mathrm{CN}$ (1:1). Absolute fluorescence quantum yields were determined in $\mathrm{PBS} / \mathrm{CH}_{3} \mathrm{CN}(1: 1)$ by means of an integrating sphere (Horiba Jobin-Yvon). All spectroscopic experiments were carried out in triplicate.

Photoactivation of Compounds 1 and 2 in Solution. Photoactivation of compounds 1 and 2 was performed in an RMR-600 photoreactor (Rayonet) equipped with eight RMR-3000 Å ultraviolet light bulbs. For fluorescence analysis, solutions of 1 and 2 were prepared in $\mathrm{PBS} / \mathrm{CH}_{3} \mathrm{CN}(1: 1,0.25 \mu \mathrm{M})$ and transferred to a quartz fluorescence cuvette (Thorlabs) to a final volume of $2.5 \mathrm{~mL}$. The solutions were irradiated $\left(32 \mathrm{~W}, 25^{\circ} \mathrm{C}\right)$ for a total of $25 \mathrm{~min}$. Fluorescence intensity was measured $\left(\lambda_{\mathrm{ex}}=\right.$ $540 \mathrm{~nm}$ and $\left.\lambda_{\mathrm{em}}=550-650 \mathrm{~nm}\right)$ before activation $(t=0 \mathrm{~min})$ and at fixed time intervals $(t=1,2,3,4,5,6,7,8,9,10,15,20$ and 25 
$\min )$. The data were plotted as the integrated fluorescence intensity normalized to the initial intensity $(I=1)$. For the procedure to determine quantum yields of photoactivation, see the SI.

Cell Culture. HeLa cells (ATCC CCL2) were grown in Dulbecco's Modified Eagle Medium (DMEM) supplemented with fetal bovine serum (FBS, 10\%) and penicillin-streptomycin $(0.1 \%)$ at $37{ }^{\circ} \mathrm{C}$ in a $95 \%$ humidity atmosphere under $5 \% \mathrm{CO}_{2}$ environment. Cells were grown to $90 \%$ confluence and seeded onto Nunc Lab-Tek II chambered cover glass plates $48 \mathrm{~h}$ prior to imaging experiments. Before imaging, the growth medium was removed and the cells were washed with PBS (0.5 mL). Cells were subsequently incubated for the indicated time with $0.5 \mathrm{~mL}$ of imaging medium (Fluorobrite, Thermo Fisher Scientific) containing the fluorescent probe $(10 \mu \mathrm{M})$ and imaged in the same medium.

Cell Viability Assay. The viability of HeLa cells was determined using methylthiazoluldiphenyl-tetrazolium bromide (MTT). Two 96-well plates were prepared with each well containing 9000 cells previously grown to $90 \%$ confluency and attached during a $24 \mathrm{~h}$ incubation in growth medium. The cells were treated with compound 1 at different concentrations (Figure S22). Solutions of compound $1(<110 \mu \mathrm{M})$ were prepared in DMSO with final concentration of $0.25 \%$ DMSO in growth medium. From the resulting solution, $5 \mu \mathrm{L}$ were added to each well containing the aforementioned cells in growth medium. Positive controls were prepared with DMSO $(0.25 \%, 100 \%$ viability). After addition of compound 1 , one plate was irradiated in a transilluminator $(410 \mathrm{~nm})$ for $30 \mathrm{~min}$, while the second plate was kept in the dark. After $72 \mathrm{~h}$ incubation at $37^{\circ} \mathrm{C}_{\text {and }} \mathrm{CO}_{2}(5 \%)$, the cells were treated with $10 \%$ MTT solution $\left(5 \mathrm{~g} \mathrm{~L}^{-1}\right)$ in imaging medium (Fluorobrite) and incubated for $3 \mathrm{~h}$. The supernatant was carefully removed and replaced with 2-propanol $(200 \mu \mathrm{L})$. The plates were shaken at $450 \mathrm{rpm}$ in a microplate shaker (VWR) and the absorbance of each well was read out with a plate reader (SPARK 10M, TECAN). Duplicates of triplicates were sampled for every concentration. Absolute $\mathrm{IC}_{50}$ values were determined with Prism 6 (GraphPad) and error values were based on standard deviations.

Confocal Fluorescence Microscopy. Fluorescent images of cells were taken using a Nikon Eclipse Ti Light Microscope equipped with a Yokogawa spinning-disk confocal scanner unit CSU-W1-T2, two sCMOS cameras (Orca Flash 4.0 V2) and a LUDLPrecision2 stage with a piezo focus. Diode-pumped solid-state lasers (DPSS) were used as light sources: $405 \mathrm{~nm}(120 \mathrm{~mW}$, $20 \%$ laser power), $445(100 \mathrm{~mW}, 40 \%)$ and $561 \mathrm{~nm}(200 \mathrm{~mW}, 20 \%)$. The exposure time, acquisition time and laser powers were kept constant unless stated otherwise. All images were collected using a 100x CFI Apo TIRF (NA = 1.49) objective with oilimmersion. Emitted light was filtered using the following filters: BP 450/50, BP 470/24, and BP 605/52. The microscope was operated using VisiVIEW (Metamorph) software. Quantification of fluorescence intensity was carried out using Fiji (ImageJ 1.5d, $\mathrm{NIH}$ ) or ICY (GPLv3, Institute Pasteur). The cell body was selected as the region of interest (ROI) and the integrated intensity within the ROI was measured. An additional ROI of the same size and shape was used to obtain the integrated intensity of the background (region with no cell). The background intensity was subtracted from that of the cell-containing ROI. Significant outliers were identified by the ROUT method $(\mathrm{Q}=1 \%)^{31}$ and excluded from further analysis. All data sets were analyzed by unpaired, two-tailed, Student's t-test. The statistical analyses were carried out using Prism 6 (GraphPad). Error bars represent standard deviation, $P$ values are given and results are depicted as mean.

Inhibition of Human Carboxylesterases hCEI and hCEII. Carboxylesterase inhibitors 9 and 10 were synthesized according to the reported procedures. ${ }^{22,23} \mathrm{DMSO}$ stock solutions $(1 \mathrm{mM})$ were prepared. Inhibition experiments in solution were carried out in black 96-well plates with transparent bottom (VITARIS AG) for compound $1(10 \mu \mathrm{M})$. Different samples containing 1, hCEII (Sigma, final concentration $\left.10 \mu \mathrm{g} \mathrm{m}^{-1}\right)$, and inhibitor $9(10 \mu \mathrm{M})$ were prepared (Figure S19). The plate was irradiated with $410 \mathrm{~nm}$ light (LED trans-illuminator) for $30 \mathrm{~min}$. Dark (non-irradiated) solutions containing compound 1 were added to the plate after irradiation. Fluorescence intensities were measured with a plate reader coupled to a fluorimeter (Horiba Jobin-Yvon). Triplicates of each sample were measured and the average values were reported with their respective standard deviation (Figure S19). For experiments in live cells, HeLa cells were incubated for $30 \mathrm{~min}(10 \mu \mathrm{M})$ in growth medium followed by washing with PBS. Probes 1 and 2 were incubated for $10 \mathrm{~min}(100 \mathrm{nM})$ in fresh imaging medium prior to imaging. Pictures were taken at $561 \mathrm{~nm}$ excitation with a $500 \mathrm{~ms}$ exposure time prior to photoactivation. Photoactivation of the dye was carried out with the $405 \mathrm{~nm}$ laser with $1 \mathrm{~s}$ irradiation time. Pictures after photoactivation were taken at $561 \mathrm{~nm}$ excitation with a $500 \mathrm{~ms}$ exposure time.

Intracellular Diffusion Experiment. Cells were incubated with compound $1(10 \mu \mathrm{M})$ for $10 \mathrm{~min}$. Without any further washing step, an ROI was selected for irradiation using the FRAP (fluorescence recovery after photobleaching) module available in VisiVIEW software. A time lapse imaging experiment was recorded in which the FRAP pulse (405 nm, $120 \mathrm{~mW}, 20 \%, 1 \mathrm{~s})$ was applied.

Super-Resolution Microscopy. HeLa cells were plated in phenol red-free growth medium (Gibco). Cells were washed with PBS $(0.5 \mathrm{~mL})$ before imaging and refilled with imaging medium $(0.25 \mathrm{~mL})$. Compound $1(1 \mu \mathrm{M}$ in imaging medium) was added to the cells within the first $10 \mathrm{~s}$ of acquisition (for details see below). For inhibition experiments, cells were incubated with compound 9 or 10 (10 $\mu \mathrm{M}$ in imaging medium) $30 \mathrm{~min}$ prior to imaging and the same imaging procedure was followed. Cells were imaged using a Nikon N-STORM microscope (Nikon, UK Ltd.) equipped with an SR Apochromat TIRF 100x 1.49 N.A. oil immersion objective lens. Activation and excitation lasers with wavelengths of $\lambda=405 \mathrm{~nm}$ and $\lambda=561 \mathrm{~nm}$ illuminated the sample in HILO mode. Fluorescence was detected with an iXon DU897 (Andor) EM-CCD camera $\left(16 \times 16 \mu \mathrm{m}^{2}\right.$ pixel size) with an exposure time of $10 \mathrm{~ms}$ for the laser excitation. An in-built focus-lock system was used to prevent axial drift of the sample during data acquisition. The emission was collected and passed through a laser QUAD filter set for TIRF applications (Nikon CNSTORM QUAD 405/488/561/647) comprising laser clean-up, dichroic and emission filters.

The laser excitation was at $561 \mathrm{~nm}$, with a peak power density of $1.2 \mathrm{~kW} \mathrm{~cm}^{-2}$. Photoconversion was achieved with $100 \mu \mathrm{s}$-long activation pulses of $405 \mathrm{~nm}$ laser of $46 \mathrm{~W} \mathrm{~cm} \mathrm{c}^{-2}$ peak power density, which were directed to the sample every $1 \mathrm{~s}$. The pulses were synchronized with the excitation laser using a DAQ Board and a PXI 1033 controller (National Instruments). Typically, 30,000 camera frames were recorded. From each image stack, a reconstructed super-resolved image was generated as described below.

Single-Molecule Localization and Image Analysis. Molecules were localized using the ThunderSTORM plugin of Fiji (ImageJ)..$^{32}$ Signals were detected searching the local intensity maxima in each frame, which were fit using an integrated Gaussian point spread function. Localizations with a localization precision above $40 \mathrm{~nm}$ and a sigma above $250 \mathrm{~nm}$ were filtered out. The first $15 \mathrm{~s}$ (1500 frames) were omitted from the analyses. Particle tracking and calculation of diffusion coefficients were performed 
using DiaTrack. ${ }^{27}$ The following parameters were used for particle tracking: maximum particle jump of 6 pixels $(0.48 \mu \mathrm{m})$ between frames and a minimum track length of 5 frames . A "birth" map of the tracks with diffusion coefficients $\left(D<20 \mu \mathrm{m}^{2} \mathrm{~s}^{-1}\right)$ was exported as a text file and cluster (segmentation) analysis was performed using SR-Tesseler software. ${ }^{29}$ In brief, Voronoï tessellation was applied to the image. Objects were defined as regions that enclose Voronoi polygons in which the local density $\mathcal{\delta}_{i}$ (particles per unit of area) is larger than twice the average density of the whole image $(2 \delta)$. Clusters were defined as regions within the objects in which the density of molecules was higher than the average density of the object.

\section{ASSOCIATED CONTENT}

\section{Supporting Information}

Supporting figures and schemes, synthetic procedures, spectroscopic characterization of all compounds, single-crystal x-ray diffraction methods and data, and NMR spectra. The Supporting Information is available free of charge on the ACS Publications website.

Crystallographic information of compounds 1, 5, 6, S3, and S4 (CIF)

Supporting Information (PDF)

Movie S1 of intracellular diffusion after photoactivation (AVI)

\section{AUTHOR INFORMATION}

\section{Corresponding Author}

* pablo.rivera-fuentes@org.chem.ethz.ch

\section{Author Contributions}

†. A. H. and Z. T. contributed equally.

\section{Notes}

The authors declare no competing financial interests.

\section{ACKNOWLEDGMENT}

This work was supported by ETH Zurich (grant ETH-02 16-1 to P. R.-F.). All microscopy work was carried out at the Scientific Center for Optical and Electron Microscopy (ScopeM) at ETH Zurich. We thank Dr. Giovanni Bassolino (ETH Zurich) for assistance measuring quantum yields of photoactivation, Dr. Pascal Vallotton (ETH Zurich) for advise regarding particle tracking and Dr. Szymon Stoma (ETH Zurich) for help with colocalization analysis.

\section{REFERENCES}

(1) Abbe, E. Arch. Mikrosk. Anat. 1873, 9, 413.

(2) Hell, S. W.; Sahl, S. J.; Bates, M.; Zhuang, X.; Heintzmann, R.; Booth, M. J.; Bewersdorf, J.; Shtengel, G.; Hess, H.; Tinnefeld, P.; Honigmann, A.; Jakobs, S.; Testa, I.; Cognet, L.; Lounis, B.; Ewers, H.; Davis, S. J.; Eggeling, C.; Klenerman, D.; Willig, K. I.; Vicidomini, G.; Castello, M.; Diaspro, A.; Cordes, T. J. Phys. D Appl. Phys. 2015, 48, 443001.

(3) Rust, M. J.; Bates, M.; Zhuang, X. W. Nat. Methods 2006, 3, 793.

(4) Betzig, E.; Patterson, G. H.; Sougrat, R.; Lindwasser, O. W.; Olenych, S.; Bonifacino, J. S.; Davidson, M. W.; LippincottSchwartz, J.; Hess, H. F. Science 2006, 313, 1642.

(5) Lippincott-Schwartz, J.; Patterson, G. H. Trends Cell Biol. 2009, 19, 555.

(6) Chozinski, T. J.; Gagnon, L. A.; Vaughan, J. C. FEBS Lett. 2014, 588, 3603

(7) Sauer, M.; Heilemann, M. Chem. Rev. 2017, 117, 7478.

(8) von Diezmann, A.; Shechtman, Y.; Moerner, W. E. Chem. Rev. 2017, 117, 7244.

(9) Specht, E. A.; Braselmann, E.; Palmer, A. E. Annu. Rev. Physiol. 2017, 79, 93.

(10) Carter, K. P.; Young, A. M.; Palmer, A. E. Chem. Rev. 2014, 114, 4564.

(11) Aron, A. T.; Ramos-Torres, K. M.; Cotruvo Jr., J. A.; Chang, C. J. Acc. Chem. Res. 201 5, 48, 2434.

(12) Chan, J.; Dodani, S. C.; Chang, C. J. Nat. Chem. 2012, 4, 973.

(13) Hou, J.-T.; Ren, W. X.; Li, K.; Seo, J.; Sharma, A.; Yu, X.-Q.; Kim, J. S. Chem. Soc. Rev. 2017, 46, 2076.

(14) Kaur, A.; Kolanowski, J. L.; New, E. J. Angew. Chem. Int. Ed. 2016, 55, 1602.

(15) Mo, G. C. H.; Ross, B.; Hertel, F.; Manna, P.; Yang, X.; Greenwald, E.; Booth, C.; Plummer, A. M.; Tenner, B.; Chen, Z.; Wang, Y.; Kennedy, E. J.; Cole, P. A.; Fleming, K. G.; Palmer, A.; Jimenez, R.; Xiao, J.; Dedecker, P.; Zhang, J. Nat. Methods 2017, 14, 427.

(16) Ross, M. K.; Crow, J. A. J. Biochem. Mol. Toxicol. 2007, 21, 187.

(17) Belov, V. N.; Wurm, C. A.; Boyarskiy, V. P.; Jakobs, S.; Hell, S. W. Angew. Chem. Int. Ed. 2010, 49, 3520

(18) Belov, V. N.; Mitronova, G. Y.; Bossi, M. L.; Boyarskiy, V. P.; Hebisch, E.; Geisler, C.; Kolmakov, K.; Wurm, C. A.; Willig, K. I.; Hell, S. W. Chem. Eur.J. 2014, 20, 13162.

(19) Grimm, J. B.; English, B. P.; Choi, H.; Muthusamy, A. K.; Mehl, B. P.; Dong, P.; Brown, T. A.; Lippincott-Schwartz, J.; Liu, Z.; Lionnet, T.; Lavis, L. D. Nat. Methods 2016, 13, 985.

(20) Hirabayashi, K.; Hanaoka, K.; Takayanagi, T.; Toki, Y.; Egawa, T.; Kamiya, M.; Komatsu, T.; Ueno, T.; Terai, T.; Yoshida, K.; Uchiyama, M.; Nagano, T.; Urano, Y. Anal. Chem. 2015, 87, 9061.

(21) Dickinson, B. C.; Huynh, C.; Chang, C. J. J. Am. Chem. Soc. 2010, 132, 5906.

(22) Inagaki, R.; Ninomiya, M.; Tanaka, K.; Koketsu, M. ChemMedChem 2015, 10, 1413.

(23) Hatfield, M. J.; Chen, J.; Fratt, E. M.; Chi, L.; Bollinger, J. C.; Binder, R. J.; Bowling, J.; Hyatt, J. L.; Scarborough, J.; Jeffries, C.; 
Potter, P. M. J. Med. Chem. 2017, 60, 1568 .

(24) Tokunaga, M.; Imamoto, N.; Sakata-Sogawa, K. Nat. Methods 2008, 5, 159.

(25) Manley, S.; Gillette, J. M.; Patterson, G. H.; Shroff, H.; Hess, H. F.; Betzig, E.; Lippincott-Schwartz, J. Nat. Methods 2008, 5 , 155 .

(26) Mastro, A. M.; Babich, M. A.; Taylor, W. D.; Keith, A. D. Proc. Natl. Acad. Sci. U. S. A. 1984, 81, 3414

(27) Vallotton, P.; Olivier, S. Microsc. Microanal. 2013, 19, 451.

(28) Knight, J. D.; Lerner, M. G.; Marcano-Velázquez, J. G.; Pastor, R. W.; Falke, J. J. Biophys. J. 2010, 99,2879

(29) Levet, F.; Hosy, E.; Kechkar, A.; Butler, C.; Beghin, A.; Choquet, D.; Sibarita, J.-B. Nat. Methods 2015, $12,1065$.

(30) Tang, A.-H.; Chen, H.; Li, T. P.; Metzbower, S. R.; MacGillavry, H. D.; Blanpied, T. A. Nature 2016, 536, 210.

(31) Motulsky, H. J.; Brown, R. E. BMC Bioinformatics 2006, 7, 123.

(32) Ovesný, M.; Křǐžek, P.; Borkovec, J.; Švindrych, Z.; Hagen, G. M. Bioinformatics 2014, 30, 2389. 